\title{
Newborn screening for homocystinurias: Recent recommendations versus current practice
}

Rebecca Keller ${ }^{1,2}$ | Petr Chrastina ${ }^{3}$ | Markéta Pavlíková ${ }^{3,4}$ | Sofía Gouveia ${ }^{5}$ |

Antonia Ribes $^{6}$ । Stefan Kölker ${ }^{7}$ | Henk J. Blom ${ }^{8}$ | Matthias R. Baumgartner ${ }^{1,2}$ ।

Josef Bártl $^{3}$ | Carlo Dionisi-Vici ${ }^{9}$ | Florian Gleich ${ }^{7}$ | Andrew A. Morris ${ }^{10}$ | Viktor Kožich ${ }^{3}$ |

Martina Huemer ${ }^{1,2,11}$ । and individual contributors of the European Network and Registry for

Homocystinurias and Methylation Defects (E-HOD) | Ivo Barić ${ }^{12}$ | Tawfeq Ben-Omran ${ }^{13}$ |

Javier Blasco-Alonso $^{14}$ | Maria A. Bueno Delgado ${ }^{15}$ | Claudia Carducci $^{16}$ |

Michela Cassanello $^{17}$ | Roberto Cerone ${ }^{18}$ | Maria Luz Couce ${ }^{5}$ | Ellen Crushell ${ }^{19}$ |

Carmen Delgado Pecellin ${ }^{15}$ | Elena Dulin ${ }^{20}$ | Mercedes Espada $^{21}$ | Giulio Ferino ${ }^{22}$ |

Ralph Fingerhut $^{1,23}$ | Immaculada Garcia Jimenez ${ }^{24}$ | Immaculada Gonzalez Gallego ${ }^{25}$ |

Yolanda González-Irazabal $^{26}$ ～Gwendolyn Gramer ${ }^{7}$ ｜ Maria Jesus Juan Fita ${ }^{25}$ ।

Eszter Karg ${ }^{27}$ | Jeanette Klein ${ }^{28}$ | Vassiliki Konstantopoulou ${ }^{29}$ | Giancarlo la Marca ${ }^{30,31}$ |

Elisa Leão Teles ${ }^{32}$ | Vincenzo Leuzzi ${ }^{33}$ | Franco Lilliu ${ }^{22}$ | Rosa Maria Lopez ${ }^{6}$ ।

Allan M. Lund ${ }^{34}$ | Philip Mayne ${ }^{35}$ | Silvia Meavilla ${ }^{36}$ | Stuart J. Moat ${ }^{37}$ |

Jürgen G. Okun ${ }^{7}$ | Elisabeta Pasquini (DECEASED) ${ }^{38}$ | Consuélo Carmen Pedron-Giner ${ }^{39}$ |

Gabor Zoltan Racz ${ }^{27}$ | Maria Angeles Ruiz Gomez ${ }^{40}$ | Laura Vilarinho $^{41}$ ।

Raquel Yahyaoui $^{42}$ | Moja Zerjav Tansek ${ }^{43}$ | Rolf H. Zetterström ${ }^{44,45}$ | Maximilian Zeyda ${ }^{29}$

\footnotetext{
${ }^{1}$ Division of Metabolism and Children's Research Center, University Children's Hospital Zürich, Zürich, Switzerland

${ }^{2}$ radiz-Rare Disease Initiative Zürich, Clinical Research Priority Program, University of Zürich, Zürich, Switzerland

${ }^{3}$ Department of Pediatrics and Adolescent Medicine, Charles University_First Faculty of Medicine and General University Hospital, Ke Karlovu 2, 12808 Praha 2, Czech Republic

${ }^{4}$ Department of Probability and Mathematical Statistics, Charles University_Faculty of Mathematics and Physics, Prague, Czech Republic

${ }^{5}$ Unit of Diagnosis and Treatment of Congenital Metabolic Diseases, S. Neonatology, Department of Pediatrics, Hospital Clínico Universitario de Santiago de Compostela, CIBERER, Health Research Institute of Santiago de Compostela (IDIS), Santiago de Compostela, Spain

${ }^{6}$ Division of Inborn Errors of Metabolism, Department of Biochemistry and Molecular Genetics, Hospital Clinic de Barcelona, CIBERER, Barcelona, Spain

${ }^{7}$ Division of Neuropaediatrics and Metabolic Medicine, Centre for Paediatric and Adolescent Medicine, University Hospital Heidelberg, Heidelberg, Germany

${ }^{8}$ Department of Internal Medicine, VU Medical Center, Amsterdam, The Netherlands

${ }^{9}$ Division of Metabolism, Bambino Gesù Children's Research Hospital, Rome, Italy

${ }^{10}$ Manchester Centre for Genomic Medicine, Manchester University Hospitals NHS Trust, Manchester, UK

${ }^{11}$ Department of Paediatrics, Landeskrankenhaus Bregenz, Bregenz, Austria
}

Abbreviations: C2, Acetylcarnitine; C3, Propionylcarnitine; C17, Heptadecanoylcarnitine; CBSD, Cystathionine beta-synthase deficiency; CLIR, Collaborative Laboratory Integrated Reports; cbl, Cobalamin; cRMD, Combined remethylation disorder; DBS, Dried blood spots; E-HOD, European Network and Registry for Homocystinurias and Methylation Defects; iRMD, Isolated remethylation disorder; MATI/IIID, Methionine adenosyltransferase I/III deficiency; MMA, Methylmalonic acid;
Met, Methionine; MoM, Multiples of the median; MTHFRD, Methylenetetrahydrofolate reductase deficiency; NBS, Newborn screening; Phe, Phenylalanine; RMD, Remethylation defect; ROC, Receiver operating characteristic; tHcy, Total homocysteine.

R. Keller, P. Chrastina, V. Kožich and M. Huemer contributed equally to this work. 


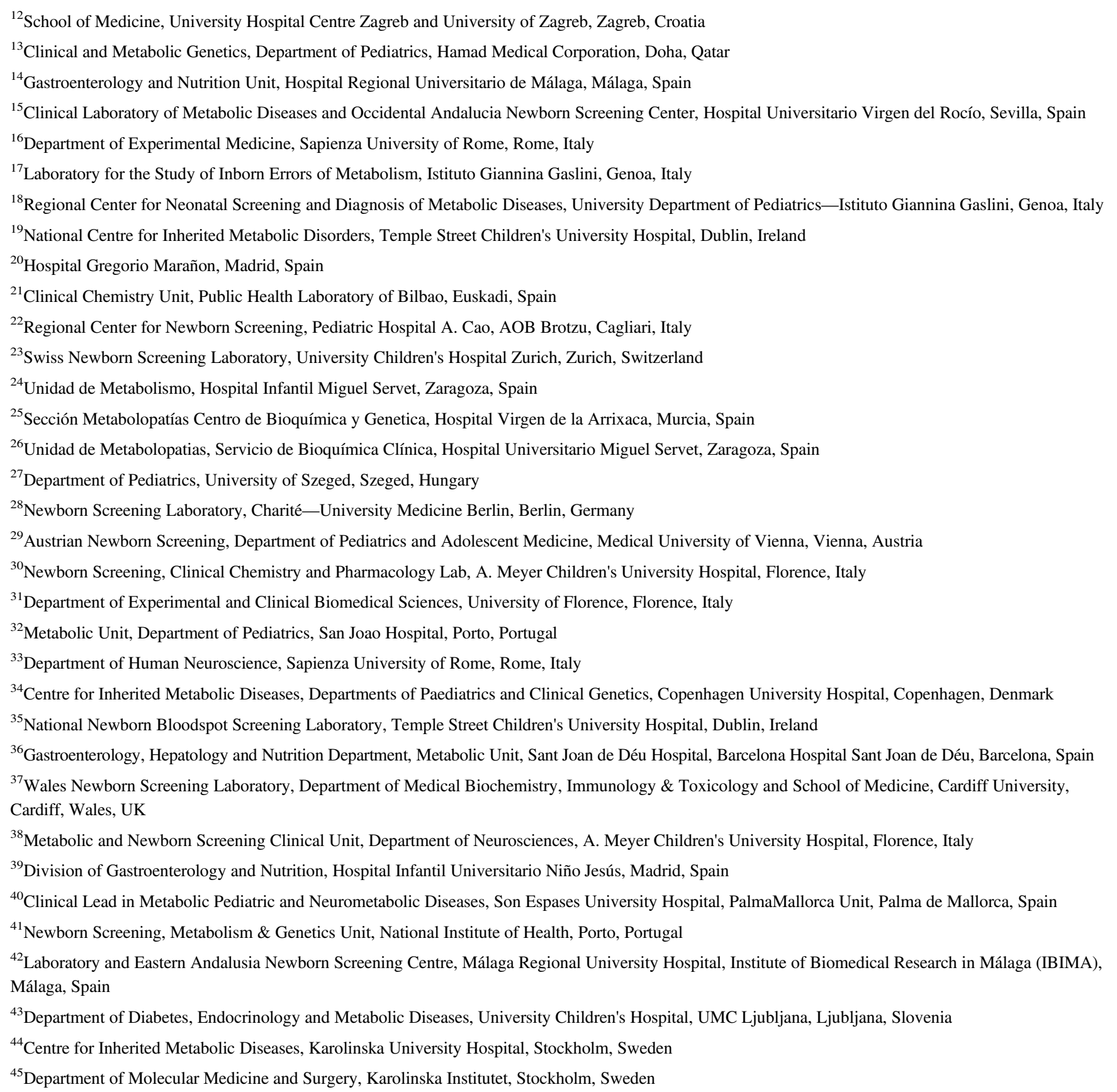

\section{Correspondence}

V. Kožich, Department of Pediatrics and Adolescent Medicine, Charles UniversityFirst Faculty of Medicine and General University Hospital, Ke Karlovu 2, 128 08 Praha 2, Czech Republic. Email: viktor.kozich@vfn.cz M. Huemer, Department of Paediatrics, Carl-Pedenz-Str.2, Landeskrankenhaus Bregenz, Austria. Email: martina.huemer@lkhb.at; martina.huemer@kispi.uzh.ch

Communicated by: Piero Rinaldo

\begin{abstract}
Purpose: To assess how the current practice of newborn screening (NBS) for homocystinurias compares with published recommendations.

Methods: Twenty-two of 32 NBS programmes from 18 countries screened for at least one form of homocystinuria. Centres provided pseudonymised NBS data from patients with cystathionine beta-synthase deficiency (CBSD, $n=19$ ), methionine adenosyltransferase I/III deficiency (MATI/IIID, $n=28$ ), combined remethylation disorder (cRMD, $n=56$ ) and isolated remethylation disorder (iRMD), including methylenetetrahydrofolate reductase deficiency (MTHFRD) $(n=8)$. Markers and decision limits were converted to multiples of the median (MoM) to allow comparison between centres.
\end{abstract}


Funding information

Mayo Clinic; Charles University; Ministry of Health of the Czech Republic
Results: NBS programmes, algorithms and decision limits varied considerably. Only nine centres used the recommended second-tier marker total homocysteine (tHcy). The median decision limits of all centres were $\geq 2.35$ for high and $\leq 0.44$ MoM for low methionine, $\geq 1.95$ for high and $\leq 0.47 \mathrm{MoM}$ for low methionine/phenylalanine, $\geq 2.54$ for high propionylcarnitine and $\geq 2.78 \mathrm{MoM}$ for propionylcarnitine/acetylcarnitine. These decision limits alone had a $100 \%, 100 \%, 86 \%$ and $84 \%$ sensitivity for the detection of CBSD, MATI/IIID, iRMD and cRMD, respectively, but failed to detect six individuals with cRMD. To enhance sensitivity and decrease second-tier testing costs, we further adapted these decision limits using the data of 15000 healthy newborns.

Conclusions: Due to the favorable outcome of early treated patients, NBS for homocystinurias is recommended. To improve NBS, decision limits should be revised considering the population median. Relevant markers should be combined; use of the postanalytical tools offered by the CLIR project (Collaborative Laboratory Integrated Reports, which considers, for example, birth weight and gestational age) is recommended. tHcy and methylmalonic acid should be implemented as second-tier markers.

\section{1 | INTRODUCTION}

Homocystinurias are rare genetic diseases caused by deficient activity of enzymes involved in the metabolism of sulfur amino acids or of the related B vitamins. Although these diseases are aetiologically and clinically heterogeneous, they share the biochemical feature of elevated concentrations of homocyst(e)ine in blood and urine. This study focuses on classical homocystinuria or cystathionine beta-synthase deficiency (CBSD), the combined remethylation disorder (cRMD), cblC, cblD-MMA-Hcy, cblF, cblJ, and the isolated remethylation disorder (iRMD), cblD-Hcy, cblE, cblG, and methylenetetrahydrofolate reductase (MTHFR) deficiency. Although methionine adenosyltransferase I/III deficiency (MATI/IIID) is generally not characterized by homocystinuria, it was included in this study because its detection by newborn screening (NBS) is closely related to NBS for CBSD and discussed in this context. ${ }^{1}$

The clinical manifestation of untreated homocystinurias depends on the affected gene and the severity of mutations. Often, cognitive impairment, seizures, white matter and ocular abnormalities, connective tissues involvement and thromboembolism are presenting signs. Details on the clinical features, diagnostic and therapeutic strategies, and efficacy of treatment were recently reviewed. ${ }^{2,3}$ The evidence discussed in these and other publications ${ }^{4-12}$ indicates that patients with homocystinurias may benefit from early treatment. A small window of opportunity for efficient intervention-especially in RMD—highlights the need for timely diagnosis, preferably by NBS.
Ideally, NBS programmes should reliably detect patients suffering from a well-characterized disorder, in which early treatment is beneficial, using high quality, economically feasible screening tests. ${ }^{13-15}$ In the past several decades, different NBS strategies for the homocystinurias have been developed. Several recent publications have proposed to adopt two-tier screening strategies. ${ }^{2,16-20}$ These approaches recommended to assess in a first step the primary markers: (a) elevated methionine (Met) and/or methionine-tophenylalanine (Met/Phe) ratio for CBSD; (b) low Met and/or Met/Phe levels for RMD; and (c) elevated propionylcarnitine (C3), propionyl/acetylcarnitine $(\mathrm{C} 3 / \mathrm{C} 2)$ ratio and possibly $\mathrm{C} 3 / \mathrm{Met}$ or heptadecanoylcarnitine for $\mathrm{CRMD}^{2,16,21}$ from dried blood spots (DBS). Subsequently, the second-tier markers total homocysteine (tHcy) for all homocystinurias and methylmalonic acid (MMA) for cRMD should be analyzed in the small number (approximately 1\%) of DBS with abnormal concentrations of primary markers.

The establishment of optimal decision thresholds is among the most challenging tasks for NBS programmes. Decision limits closer to the median of the marker in the population of healthy newborns increase sensitivity but, simultaneously, increase the false-positive rate, which determines the cost of second-tier testing. While decision limits more distant from the population median reduce the falsepositive rate, they may fail to detect patients.

It has been suggested that NBS centres should pool data and share experience on the NBS algorithms for homocystinurias, as proposed by Gramer et al.. ${ }^{16}$ However, the comparability of data among different NBS centres is a generic 
problem of population screening programmes, due to for example, different analytical platforms, times of sampling and decision thresholds. The use of Z-scores or multiples of the median (MoM) has been proposed to facilitate pooling data across various screening programmes. ${ }^{14,22,23}$

This work was part of the EU-funded European Network and Registry for Homocystinurias and Methylation Defects (E-HOD; http://www.e-hod.org) project and investigates the current practice of NBS for homocystinurias in 18 countries involved in the E-HOD project. We analyzed the spectrum of screened disorders, screening strategies and their conformity with recently published recommendations. ${ }^{2,16,18}$ Furthermore, this study evaluated the variation in decision limits and examined whether the use of MoM-corrected data could improve the performance of NBS for the homocystinurias.

\section{2 | METHODS}

\section{1 | Data acquisition}

E-HOD partners were invited to answer a survey addressing their regional practice of NBS for the homocystinurias with the following key questions:

1. Are homocystinurias part of your NBS panel?

2. Which algorithms do you use to detect homocystinurias?

3. Does your programme use second-tier testing for tHcy?

4. What are the median values of Met, Met/Phe ratio, C3 and $\mathrm{C} 3 / \mathrm{C} 2$ ratio markers in the population of healthy newborns in your centre?

5. Which decision limits do you use to flag the result of NBS as abnormal?

Pseudonymised data sets from 141 patients (from 23 centres) were extracted from the E-HOD registry; one additional E-HOD centre contributed data sets from ten patients not yet included into the registry $(n=151)$. Only patients younger than 10 years of age at data entry were selected to facilitate retrieval of data on NBS markers from local sources. Forty patients had to be excluded from further analyses due to missing data on the patient's marker at NBS, or medians of the local newborn population or information on centre decision limits ( $n=12$ with CBSD, $n=10$ with cRMD, $n=10$ with iRMD), or due to complete absence of NBS data in recently described very rare diseases $(n=5$ with $S$ adenosylhomocysteine hydrolase deficiency and $n=3$ with adenosine kinase deficiency), resulting in a final sample of 111 individuals. Four groups of patients were defined: CBSD $(n=19)$, MATI/IIID $(n=28)$, cRMD (cblC, cblD, cblJ) $(n=56)$ and iRMD (MTHFR, cblE, cblG) $(n=8)$. For CBSD, no subgroup classification according to pyridoxine responsiveness was attempted, as vitamin B6 response criteria from individual centres were ambiguous.

\section{2 | Calculation of MoM-corrected decision limits and marker values in patients}

To allow the comparison of data across populations and centres using different screening platforms, we calculated MoM of the Met, Met/Phe, C3 and C3/C2 decision limits reported by the NBS centres by dividing the values of each marker by its population median. The standardization of individual NBS marker values of patients with homocystinurias to MoM was performed accordingly.

\section{3 | Performance of decision limits}

To assess the performance of the highly dispersed decision limits, we calculated the range and median decision limits for each MoM-normalized marker. In a subset of patients with complete data sets, we evaluated the sensitivity of the extreme values and of the median of decision limits by calculating the proportion of patients with homocystinurias that would be detected by the respective decision limit. In addition, we analyzed the sensitivity of a combination of markers and created respective two-dimensional plots.

\subsection{Modeling sensitivity, specificity and cost- effectiveness in one NBS programme}

For this analysis, data on markers in 15000 healthy newborns from one NBS programme were combined with data of the 111 patients reported in this study. For each of the four targeted (groups of) disorders (CBSD, MATI/IIID, iRMD and cRMD), we examined how the use of specific decision limits would influence the sensitivity and specificity of the combination of markers in the model population. Firstly, we constructed two-dimensional grids for Met and Met/Phe, or $\mathrm{C} 3$ and $\mathrm{C} 3 / \mathrm{C} 2$ values for the combined pool of controls and patients. Next, we computed the sensitivity and specificity of two scenarios, that is, when both markers would be exceeding decision limits (marked as "AND" test) or when at least one of the two markers would be crossing the decision limits (marked as "OR" test). To compare the sensitivity and specificity of these marker combinations, we constructed receiver operating characteristic (ROC) curves. Finally, we used the data set to model specificity as an important determinant of either the false-positive rate or of the cost of second-tier testing by constructing contour plots of the sensitivity of markers with specificity fixed at $99 \%$ and above. The annual cost savings vs expenditure of the number of second-tier tHcy tests was calculated from the costs of tHcy analysis in DBS (approximately $€ 30.90$ per analysis) according to the 
reimbursement rate (including instrument depreciation and labour) published in the directive of the Ministry of Health of the Czech Republic on the reimbursement of healthcare procedures for 2017 (https://www.mzcr.cz/dokumenty/ vyhlaska-c/2016_13142_999_3.html).

\section{5 | Statistical methods}

Computations and model testing were performed in the statistical language and environment R (v.3.2.2), and SPSS and NCSS statistical software, respectively. Comparison of NBS markers between patients missed by NBS and those detected by NBS was carried out using Statistica Cz, version 12 (StatSoft, Inc., Tulsa, OK, USA); the Mann-Whitney $U$-test with BenjaminiHochberg correction for multiple testing was employed and significance of the false discovery rate was set at $<0.05$.

Data on the median values of markers stratified by covariates were extracted using the productivity tool "Reference Range by Covariate" on the CLIR website (https://clir.mayo. edu) on April 23, 2018. Graphs were constructed using Excel.

\section{3 | RESULTS}

\section{1 | NBS programmes for homocystinurias in surveyed countries}

Thirty-two centres from 16 European and two non-European countries contributed data on their NBS panels operating in 2016. The number of primarily targeted disorders varied considerably among countries as well as among regional programmes within countries. Twenty-one centres screened for CBSD and 15 primarily targeted cblC disease. The Italian, Czech and Spanish centres and Qatar (analyses for Qatar performed by the NBS Centre Heidelberg, Germany until 2016) also screened for iRMD (MTHFRD and/or isolated cobalamin-related remethylation defects). MATI/IIID was a primary target in Austrian, Italian and some Spanish centres. Data from the 2011 survey in 16 European countries ${ }^{24}$ and the present study cannot be easily compared due to the different grouping of disorders and countries included; however, four European countries initiated screening for CBSD and two for CRMD as the primary target in at least one centre since $2011 .^{24}$

\section{2 | NBS algorithms}

Detailed information on NBS algorithms was available for 19 centres reporting a variety of combinations and sequences of the use of Met, Met/Phe, C3 and C3/C2 markers. tHcy was used as a primary marker only in Qatar. Although recent recommendations propose so, only 9 of 24 centres adopted a two-tier strategy with tHcy as the second-tier marker. As an example of the complexity and heterogeneity of algorithms in different NBS programmes, Supplementary Figure 1 summarizes the approaches targeting the cblC defect and other cRMD.

\section{3 | Decision limits for Met, Met/Phe, C3 and $\mathrm{C} 3 / \mathrm{C2}$}

To test whether differences in decision limits among programmes are caused by different concentrations or ratios of markers in the respective normal newborn populations, we firstly compared the medians of each marker (see Figures 1 and 2). The distribution of markers in healthy newborns varied substantially among centres. Medians for Met, Met/Phe, $\mathrm{C} 3$ and $\mathrm{C} 3 / \mathrm{C} 2$ were in the range $12.8-23 \mu \mathrm{mol} / \mathrm{L}, 0.22-0.51$, $1.27-2.1 \mu \mathrm{mol} / \mathrm{L}$ and $0.057-0.18$, respectively. It is, however, of note that the dispersion of decision limits for Met, Met/Phe, $\mathrm{C} 3$ and $\mathrm{C} 3 / \mathrm{C} 2$ was even wider than the dispersion of medians, indicating a substantial lack of consensus on optimal thresholds to flag values as abnormal (Figures 1 and 2 and Supplementary Table 1).
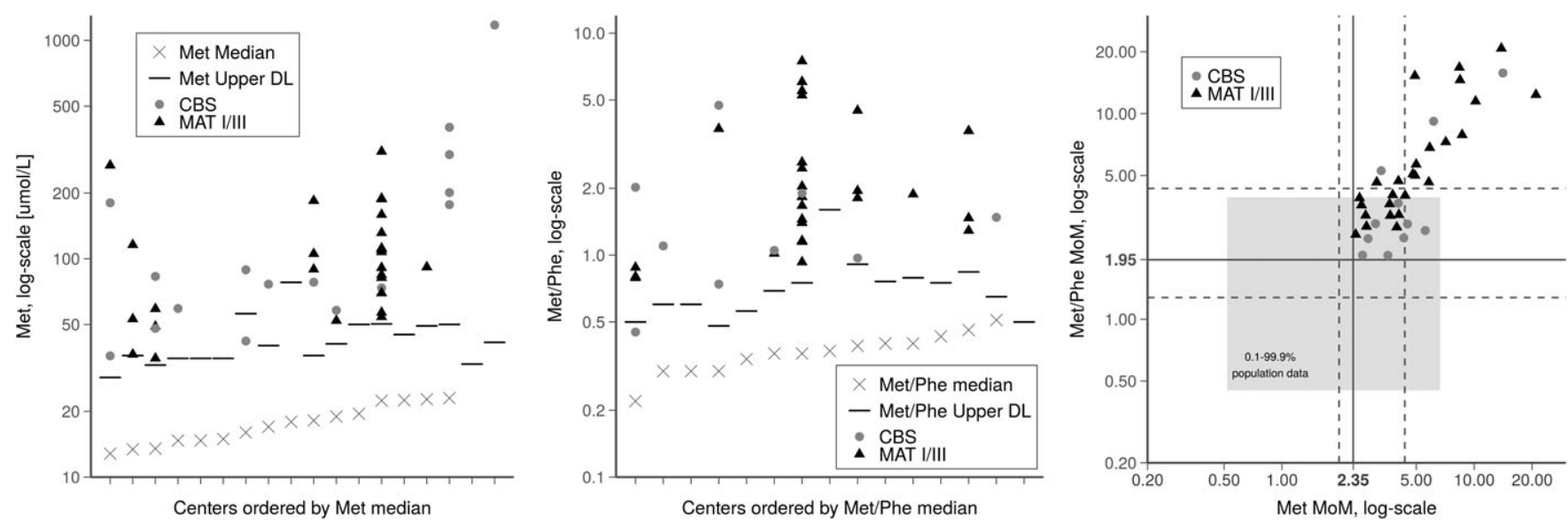

F I G U R E 1 Decision limits and marker values in patients with cystathionine beta-synthase deficiency (CBSD) and methionine adenosyltransferase I/III deficiency (MATI/IIID) 

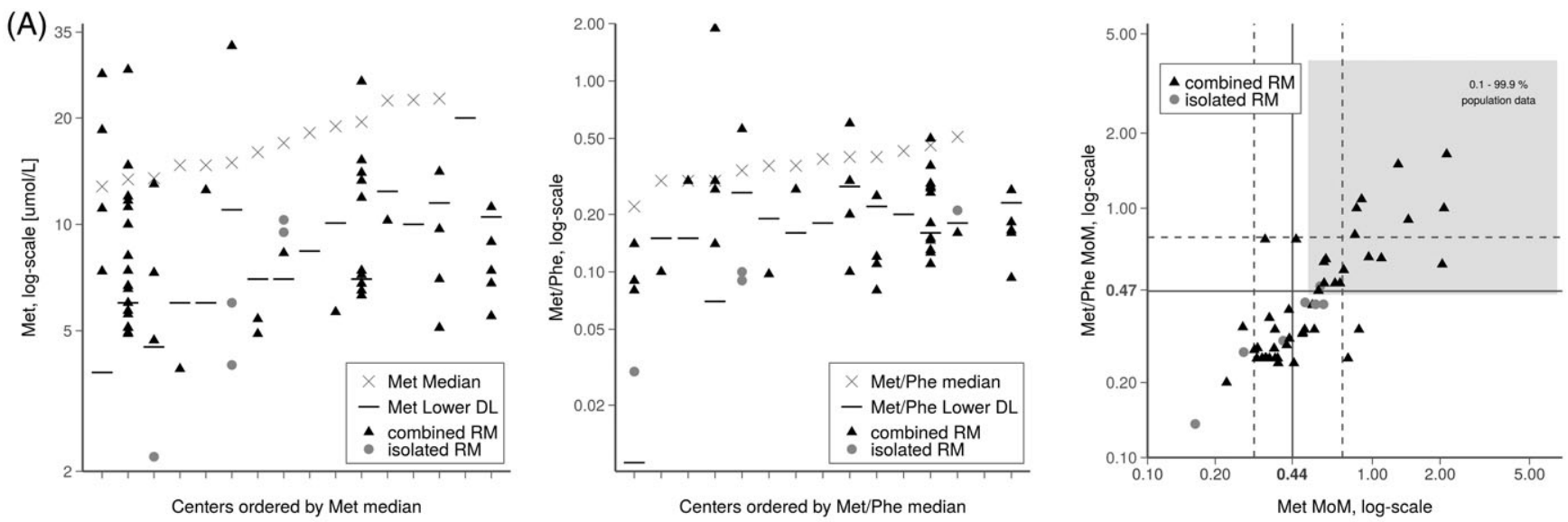

(B)
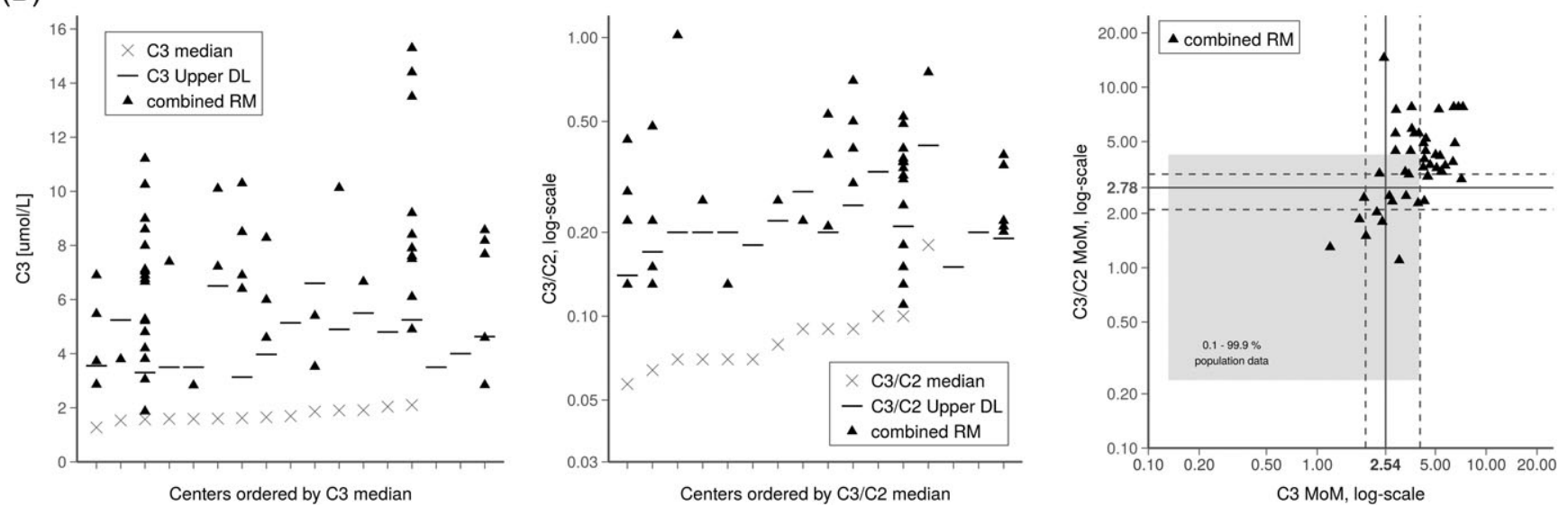

FIG URE 2 Decision limits and marker values in patients with isolated remethylation disorder (iRMD) and combined remethylation disorder (cRMD)

\section{4 | Sensitivity of decision limits to detect patients with homocystinurias}

In the subset of patients with complete data sets, we evaluated the sensitivity of decision limits.

\subsection{1 | Performance of centres' decision limits}

The proportion of patients with homocystinurias that would be detected according to the decision limits of respective centres varied considerably and clearly showed that some programmes use decision limits with unsatisfactory sensitivity (Supplementary Table 1).

Since there is no consensus on the optimal decision thresholds, we examined in the next step the sensitivity of the medians of decision limits of all programmes (as the possible best proxy of consensual values), as well as the sensitivity of combinations of markers. These medians of MoM-normalized decision limits of all centres are labeled as "suggested cut-offs" in the following paragraphs (Table 1).

\subsection{2 | Performance of median decision limits of high Met and Met/Phe to detect CBSD and MATI/IIID}

The suggested cut-offs for high Met $(\geq 2.35 \mathrm{MoM})$ or high Met/Phe ( $\geq 1.95 \mathrm{MoM})$ detected all 17 patients with CBSD and all 28 patients with MATI/IIID in this study. Congruently, the AND/OR combination of these markers detected all patients (Supplementary Tables 1 and 2 and Figure 1).

\subsection{3 | Performance of median decision limits of low Met and Met/Phe to detect iRMD}

The study cohort contained data on Met from eight and Met/Phe from seven patients with iRMD. The suggested cutoff for low Met $(\leq 0.44 \mathrm{MoM})$ detected only three of eight patients; low Met/Phe $(\leq 0.47 \mathrm{MoM})$ would detect six of seven patients. The combination of low Met AND/OR low Met/Phe would detect six of seven patients (Supplementary Tables 1, 2 and 3 and Figure 2). 
T A B L E 1 Newborn screening (NBS) panels for homocystinurias in 2016 [data provided by European Network and Registry for Homocystinurias and Methylation Defects (E-HOD) partners]

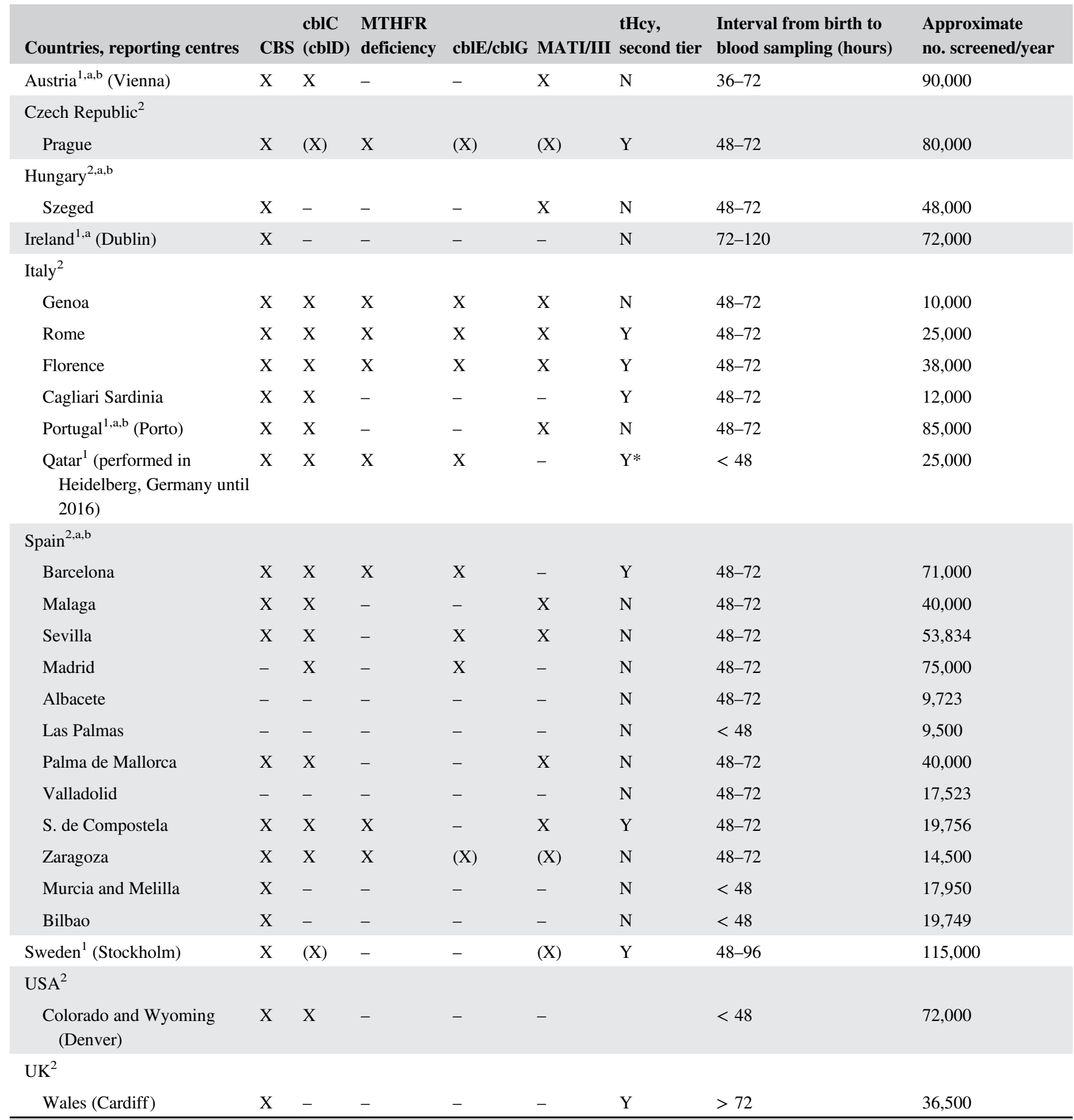

- = not primarily targeted; $\mathrm{X}=$ primary target; $(\mathrm{X})=$ secondary target (may be detected, although not targeted); $\mathrm{Y}=$ yes; $\mathrm{N}=$ no; *tHcy used as the primary marker in the Qatar NBS screening programme only.

${ }^{1}$ Single centre per country.

${ }^{2}$ More than one centre per country.

Countries with NBS for ${ }^{\mathrm{a}} \mathrm{CBS}$ or ${ }^{\mathrm{b}}$ methylmalonic acidaemia, including $\mathrm{Cbl} \mathrm{A}, \mathrm{B}, \mathrm{C}, \mathrm{D}$ defects, in place in a European survey of $2011 .{ }^{24}$

No No NBS for these disorders in: Belgium ${ }^{2, b}$ (Brussels, Flemish part); Switzerland ${ }^{1}$ (Zurich); Germany ${ }^{2}$ (Heidelberg); Slovenia $^{1}$ (Ljubljana); Croatia ${ }^{1}$ (Zagreb); Denmark $^{1, \mathrm{~b}}$ (Copenhagen); France ${ }^{2}$ (Paris). 


\subsection{4 | Performance of median decision limits of low Met and Met/Phe, and high $\mathrm{C3}$ and $\mathrm{C} 3 / \mathrm{C} 2$ to detect $\mathrm{CRMD}$}

The group of patients with cRMD with any information on decision limits consisted of 47 subjects, of which six had been missed by their original NBS programme (another cRMD patient missed by NBS was excluded from these analyses due to missing information on decision limits). Data sets (all four markers) were not complete in all patients.

The sensitivity of the suggested cut-offs for low Met $(\leq$ $0.44 \mathrm{MoM})$ and low Met/Phe $(\leq 0.47 \mathrm{MoM})$ was poor, detecting only 22 and 28 of 47 patients, respectively. In contrast, the suggested cut-offs for high C3 ( $\geq 2.54 \mathrm{MoM})$ and $\mathrm{C} 3 / \mathrm{C} 2(\geq 2.78 \mathrm{MoM})$ performed better, detecting 41 of 47 and 33 of 45 patients, respectively. The AND/OR combination of all four markers yielded slightly better results. At least one marker was outside the reference range in 43 cases. In summary, the combination of all four markers detected more patients with cRMD compared to the use of only single markers, but their performance was not sufficient to detect all patients.

In order to shed more light on the subgroup of the patients missed by their local NBS programmes, we conducted a detailed analysis of the NBS markers obtained at the time of screening. The NBS markers for cblC and cRMD in general are high $\mathrm{C} 3$ and $\mathrm{C} 3 / \mathrm{C} 2$, or low methionine and Met/Phe. Compared to patients with cblC disease detected by regional NBS programmes (data available for $n=28$ ), patients undetected by NBS (data available for $n=6$ ) exhibited significantly higher median Met (1.7 times), MoM Met (2 times), Met/Phe (1.9 times) and MOM Met/Phe (2.4-times), as well as significantly lower C3 (41\%), MoM C3 (45\%), C3/C2 (52\%) and MOM C3/C2 (53\%). These analyses demonstrate that cblC patients (or cRMD patients in general) with milder biochemical phenotype at the time of blood sampling may be missed by NBS even if consensual decision limits would be used (Supplementary Table 4).

\section{5 | Sensitivity, specificity and cost- effectiveness in a model NBS programme using median decision limits of all NBS programmes}

The analysis of specificity and sensitivity requires not only data on marker values in patients, but also data on marker distributions in the population of healthy newborns. As an example of how data from this study may be used for optimizing NBS programmes, we modeled these markers using data on Met and Met/Phe in 15000 newborns from one selected NBS programme.

The left panels in Figure 3 show the ROC curves for different markers and truth-functional operators (ie, the AND combination of two markers or the OR combination of two markers). The area under the ROC curve is over 0.99 in CBSD using either high Met AND high Met/Phe or high Met OR high Met/Phe. For cRMD, the area under the ROC curve was substantially higher for marker combinations employing high C3 AND/OR high C3/C2 (0.97 and 0.98, respectively) compared to the combination low Met AND/OR low Met/Phe (0.84 and 0.87, respectively).

The middle and right panels of Figure 3 show nomograms of specificity at different fixed sensitivities and nomograms of sensitivity at a fixed specificity of 0.99 . In this study, the nomograms were used to optimize the decision limits of a single NBS programme for homocystinurias. Lowering of the present decision limits for high Met and high Met/Phe from 2.7 to 2.56 and from 2.27 to 2.08, respectively, increased the sensitivity for detecting CBSD from 0.94 to 1.0; the number of second-tier tests increased by only $0.03 \%$, with a cost increase from $€ 4942$ to $€ 5766$ per year. For iRMD, an increase of the decision limits for low Met and low Met/Phe from 0.50 to $0.53 \mathrm{MoM}$ and from 0.55 to $0.60 \mathrm{MoM}$, respectively, maintained the same sensitivity of 0.88 ; this modification decreased substantially the number of second-tier tests of tHcy from 1.8 to $0.9 \%$ and the costs from $€ 39867$ to $€ 16639$ per year. In this analysis, the modified approach for the model-tested NBS programme yielded a sensitivity of 1 for CBSD and 0.88 for iRMD, with less demand for second-tier tHcy testing, leading to savings of $€ 22404$ per year.

\section{4 | DISCUSSION}

The long-term evidence on favorable clinical outcome in early treated patients strongly indicates that CBSD is a good candidate for NBS. ${ }^{2,3,16,18}$ NBS for RMD should at least be considered. ${ }^{2,4,8-10,25}$ MTHFR deficiency seems to be particularly responsive to early betaine treatment ${ }^{5}$ and the majority of cobalamin-related RMD have a better clinical outcome with less mortality or severe organ complications when treated early. Brain and eye disease in RMD (especially in the cblC defect), however, may progress despite treatment. The recommendations on MATI/IIID as a target condition are controversial, mostly due to the detection of large numbers of heterozygotes. ${ }^{2}$

This study involving 18 countries shows that NBS practice is not concordant with these recommendations and, despite some evolution since $2011,{ }^{24}$ NBS for the homocystinurias is not widely established.

The decision on disorders to be included into NBS programmes is only partly evidence based. The final programme setup is a political decision of states, regions and institutions, and this explains the heterogeneity among different regions and countries. In the USA, the Advisory Committee on Heritable Disorders in Newborns and Children 

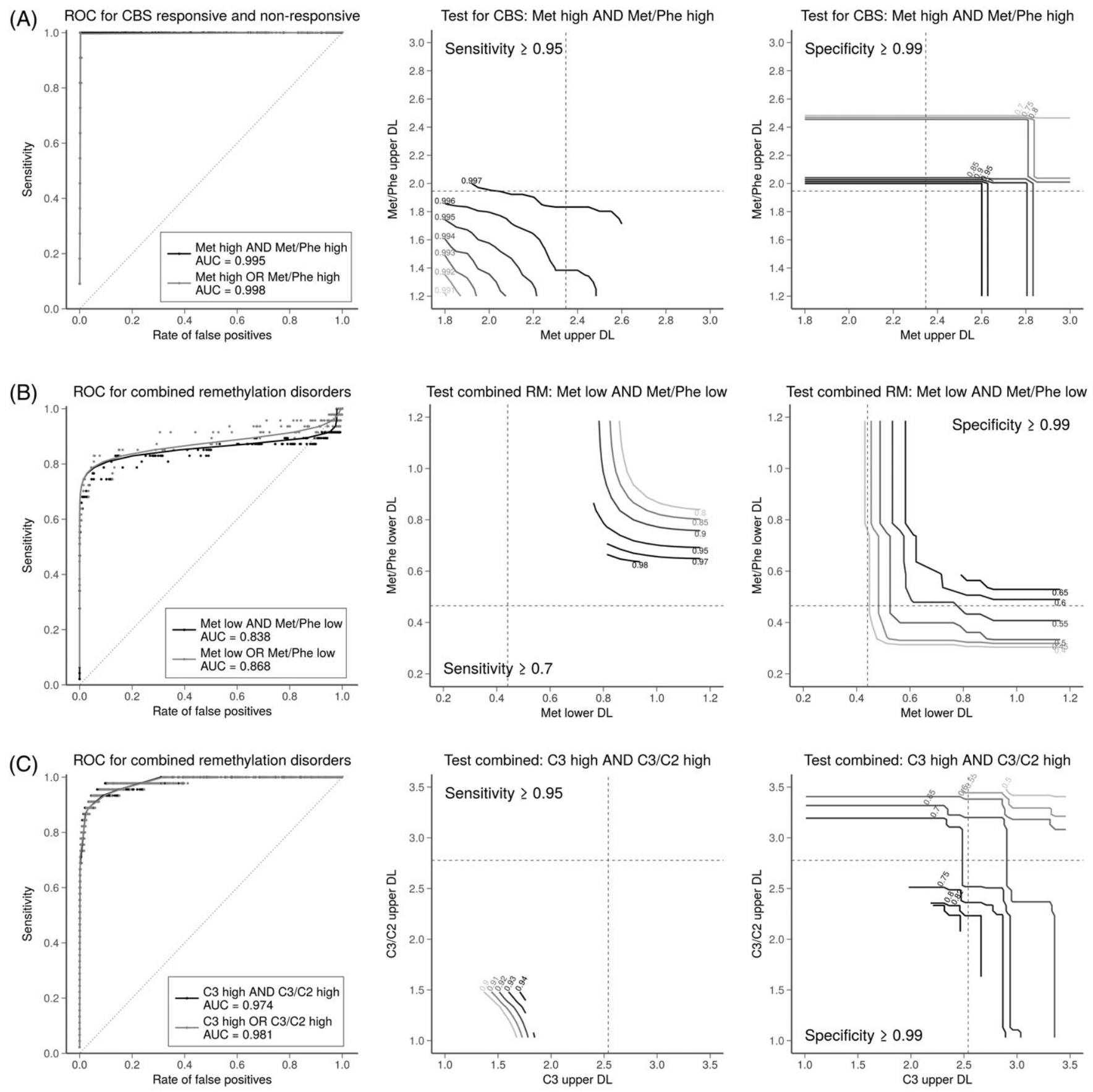

F I G U RE 3 Model of the sensitivity and specificity of markers for detecting CBSD and cRMD

(https://www.hrsa.gov/advisorycommittees/mchbadvisory/he ritabledisorders/recommendedpanel) recommended screening for CBSD as a primary target, and RMD and MATI/IIID as secondary conditions. In the EU, the absence of harmonized recommendations is reflected in the wide range from none to seven forms of homocystinuria targeted in different European NBS programmes. Interestingly, there is considerable variation in the types of homocystinuria included in NBS panels, even between regions within one country, reflecting different decision-making routes.
This study also showed a large variability in decision limits between individual programmes and demonstrated that some programmes use decision limits with suboptimal sensitivity. Only a small proportion of programmes use second-tier strategies that would allow the implementation of decision limits with higher sensitivity.

There are several reasons for the differences in decision limits between centres. Most probably, the different performance, of the various analytical programmes in place add to the heterogeneity we observe. This effect is demonstrated by 
the Centers for Disease Control and Prevention (CDC) Newborn Screening Quality Assurance Program. Data from the CDC on Set 2 July-October $2016^{26}$ show that, between seven different types of analytical platforms, the mean Met concentrations in a single DBS batch varied between 17.6 and $24.1 \mu \mathrm{mol} / \mathrm{L}$.

The variable age at sampling may be a factor, given the physiological changes in marker concentrations during the first days of life. In the Collaborative Laboratory Integrated Reports (CLIR; http://clir.mayo.edu), considerable changes of marker values within the interval of sampling after birth are apparent for Met (median $23 \mu \mathrm{mol} / \mathrm{L}$ at 24 hours and $20.1 \mu \mathrm{mol} / \mathrm{L}$ at 48 hours), Met/Phe (median 0.41 at 24 hours and 0.39 at 60 hours) and C3/C2 (median 0.075 at 48 hours and 0.081 at 60 hours). For details, see Supplementary Figure 3.

In term-born (between 37 and 41 weeks of gestation) newborns with normal weight (2500-4200 g), the influence of other covariates appears less pronounced compared to the influence of sampling time. Sex seems to be only associated with the $\mathrm{C} 3 / \mathrm{C} 2$ ratio (approximately 0.003 higher in females). Birth weight and gestational age are negatively correlated with Met and Met/Phe (eg, a decrease of approximately 0.02 for Met/Phe from the highest gestational age or weight to the lowest gestational age or weight). Furthermore, birth weight and gestational age are positively correlated with $\mathrm{C} 3$ and $\mathrm{C} 3 / \mathrm{C} 2$ (eg, $\mathrm{C} 3 / \mathrm{C} 2$ increases by approximately 0.08 and 0.09 from the highest gestational age or weight to the lowest gestational age or weight, respectively).

Newborns with lower or higher birth weight or born early/late, however, exhibit larger variability of marker values, clearly indicating the necessity of using weight- and/or gestational age-adjusted marker values (http://clir.mayo.edu ${ }^{27}$ ).

The effect of different platforms and sampling times may be reduced by using MoM. Nevertheless, in this study, the use of MoM-corrected values did not decrease the huge variability of decision limits among centres. The lack of concordance may be explained by the use of different metrics (eg, different percentiles of the reference population used as cutoffs), various degrees of acceptance of false positivity or different follow-up procedures in individual programmes.

Second-tier testing allows more sensitive decision limits to be adopted without causing a massive increase in falsepositive results. This is important as recalls lead to psychological stress for families and increased costs. ${ }^{16,28}$ Gramer et al. ${ }^{16}$ have proposed an algorithm to detect CBSD and RMD with tHcy as a second-tier test in all newborns with abnormalities in either Met (lower cut-off only) or Met/Phe (lower and upper cut-offs) or elevated $\mathrm{C} 3$ or $\mathrm{C} 3 / \mathrm{C} 2$. This approach was tested in a single centre and did not only identify patients from the local population but also patients documented in the Region 4 Collaborative Project. ${ }^{16}$ The authors show that second-tier measurement of tHcy allows adjusting the decision limits of the primary markers towards the median, which results in a smaller proportion of undetected patients. These and similar recommendations to use tHcy and/or MMA as second-tier markers ${ }^{2}$ have not yet been widely adopted in NBS practice, as demonstrated by our study. Consequently, many NBS programmes for homocystinurias have unnecessarily large numbers of false-positive results and miss the opportunity to establish more sensitive decision limits. There are currently ongoing pilot studies evaluating the use of second-tier strategies for homocystinurias and RMD, which may lead to wider application of such strategies in the future..$^{29,30}$

A most promising approach to increase both the sensitivity and specificity of NBS and to become increasingly independent from single-parameter decision limits is the use of postanalytical tools as offered by the CLIR project. These tools integrate information on larger sets of markers and correct for covariates using comprehensive statistical procedures. Unfortunately, data on the majority of the parameters used by the tools were fragmentary or not available at all in our sample, owing to the retrospective design of the E-HOD registry project.

This study has several limitations. It is not entirely representative as it exclusively includes centres participating in the E-HOD project, who may have a particular interest in homocystinurias. In addition, the retrospective nature of this study did not permit collecting uniform, complete data sets for all cases. It is also important to note that the favorable cost-effectiveness analysis was estimated from a single programme, and may not be universally applicable.

With the exception of seven cblC/cblD patients missed by NBS, all data on marker values come from patients detected by NBS. Consequently, marker values from patients with milder forms of disease are probably underrepresented in the data set, which, in turn, probably leads to a biased evaluation of decision thresholds. Unfortunately, false-negative cases are not easily detectable and the retrospective analyses of stored DBS from patients with milder or late-onset forms of homocystinurias are hampered by the instability of some markers, ethical concerns or the early destruction of DBS according to local or national policies.

\section{5 | CONCLUSIONS}

This study demonstrates that many newborn screening (NBS) programmes have not yet implemented the recent recommendations on screening for the homocystinurias. Due to the favorable outcome of early treated patients, we recommend the implementation of NBS for homocystinurias in national and regional programmes. To improve the 
performance of NBS programmes, we recommend a fourstep strategy:

1. Thorough revision of local decision limits with reference to the median of the local reference population.

2. Combination of relevant markers, as demonstrated in this study.

3. Use of the centre-adjusted postanalytical tools offered by the Collaborative Laboratory Integrated Reports (CLIR) project, which, in addition, considers covariates such as birth weight, gestational age and sampling time.

4. Implementation of total homocysteine (tHcy) and methylmalonic acid (MMA) as second-tier markers to allow for more sensitive decision limits, increased specificity and lower costs.

\section{ACKNOWLEDGEMENTS}

We thank Professor Burkhardt Seifert, Dept. of Biostatistics, University of Zürich for the statistical advice, Erica L. Wright for providing information on the Colorado and Wyoming screening programme and Marike Groenendijk (E-HOD project) for her continuous support. PCh, JB, MP and VK received institutional support from the Ministry of Health (project DRO VFN64165) and from the Charles University (project PROGRES Q26). This publication arises from the E-HOD project (Chafea grant no. December 2, 2012), which has received funding from the European Union, in the framework of the Health Programme. Many programmes cooperate closely with Piero Rinaldo and coworkers (Mayo Clinic, Rochester, Minnesota, USA) and wish to acknowledge the ongoing support provided by the R4S and CLIR initiatives (http://clir.mayo.edu).

\section{COMPLIANCE WITH ETHICAL STANDARDS}

All procedures followed were in accordance with the Helsinki Declaration of 1975 , as revised in 2000. This study was part of the E-HOD project and has ethical approval in Zürich (KEK Switzerland no. 2012-0020) and in local centres as required.

\section{Conflict of interest}

M Huemer has received research grants from Nutricia and SOBI and honorariums for lectures from Nutricia, Recordati Rare Disease Foundation, Shire and Genzyme. HJ Blom received a research grant from Orphan Europe. S Kölker receives financial support for the E-HOD registry management by the European Union, for the Cystadane Surveillance Protocol by Orphan Europe and for a pilot newborn screening study including homocystinurias and methylation defects by the
Dietmar Hopp Foundation, St. Leon-Rot, Germany. MR Baumgartner declares that the University Children's Hospital Zurich has received educational and research grants from Actelion, Genzyme and Milupa Metabolics, and receives support for the E-HOD Registry/Cystadane surveillance programme from Orphan Europe. AA Morris has received honoraria for lectures from Nutricia and Recordati Rare Disease Foundation. V Kožich declares that the Charles University-First Faculty of Medicine has received support from the Recordati Rare Disease Foundation for organizing an educational course on homocystinurias and methylation defects, and reimbursement for laboratory analyses from Orphan Technologies. C Dionisi-Vici has received research grants, speaker and consultancy honoraria from Nutricia, Medifood, SOBI and Dr. Schär Medical Nutrition. AM Lund and RH Zetterström have received grants and travel reimbursement from Orphan Europe, Nutricia and SOBI. E Crushell has received an honorarium for a lecture from Nutricia Metabolics. G la Marca has received travel reimbursement from Nutricia and research grants from Genzyme. C Pedron-Giner has received support from Vitafló-Nestlé España to attend SSIEM meetings. I Barić has received speaker's honorarium and travel support from the Recordati Rare Disease Foundation. G Gramer receives financial support for a pilot newborn screening study including homocystinurias and methylation defects by the Dietmar Hopp Foundation, St. Leon-Rot, Germany and received speaker honoraria from MetaX, Friedberg, Germany and the Recordati Rare Disease Foundation. E Pasquini has received speaker's honorarium and travel support from SANOFI Genzyme, Shire, Alexion. R Keller, P Chrastina, J Bártl, S Gouveia, A Ribes, F Gleich and M Pavlíková declare that they have no relevant conflict of interest.

\section{REFERENCES}

1. Chien YH, Abdenur JE, Baronio F, et al. Mudd's disease (MAT I/III deficiency): a survey of data for MAT1A homozygotes and compound heterozygotes. Orphanet J Rare Dis. 2015;10:99. https://doi.org/10.1186/s13023-015-0321-y4545930.

2. Huemer M, Kožich V, Rinaldo P, et al. Newborn screening for homocystinurias and methylation disorders: systematic review and proposed guidelines. J Inherit Metab Dis. 2015;38:1007-1019. https://doi.org/10.1007/s10545-015-9830-z4626539.

3. Morris AAM, Kožich V, Santra S, et al. Guidelines for the diagnosis and management of cystathionine beta-synthase deficiency. J Inherit Metab Dis. 2017;40(1):49-74.

4. Carrillo-Carrasco N, Chandler RJ, Venditti CP. Combined methylmalonic acidemia and homocystinuria, cblC type. I. Clinical presentations, diagnosis and management. J Inherit Metab Dis. 2012; 35:91-102. https://doi.org/10.1007/s10545-011-9364-y.

5. Diekman EF, de Koning TJ, Verhoeven-Duif NM, Rovers MM, van Hasselt PM. Survival and psychomotor development with early betaine treatment in patients with severe methylenetetrahydrofolate reductase deficiency. JAMA Neurol. 2014;71:188-194. https://doi.org/10.1001/jamaneurol.2013.4915. 
6. Gan-Schreier H, Kebbewar M, Fang-Hoffmann J, et al. Newborn population screening for classic homocystinuria by determination of total homocysteine from Guthrie cards. J Pediatr. 2010;156: 427-432. https://doi.org/10.1016/j.jpeds.2009.09.054.

7. Huemer M, Scholl-Bürgi S, Hadaya K, et al. Three new cases of late-onset cblC defect and review of the literature illustrating when to consider inborn errors of metabolism beyond infancy. Orphanet J Rare Dis. 2014;9:161. https://doi.org/10.1186/s13023-014-016114255922.

8. Martinelli D, Deodato F, Dionisi-Vici C. Cobalamin C defect: natural history, pathophysiology, and treatment. J Inherit Metab Dis. 2011;34:127-135. https://doi.org/10.1007/s10545-010-9161-z.

9. Weisfeld-Adams JD, Morrissey MA, Kirmse BM, et al. Newborn screening and early biochemical follow-up in combined methylmalonic aciduria and homocystinuria, cblC type, and utility of methionine as a secondary screening analyte. Mol Genet Metab. 2010; 99:116-123. https://doi.org/10.1016/j.ymgme.2009.09.008.

10. Weisfeld-Adams JD, Bender HA, Miley-Åkerstedt A, et al. Neurologic and neurodevelopmental phenotypes in young children with early-treated combined methylmalonic acidemia and homocystinuria, cobalamin C type. Mol Genet Metab. 2013;110:241-247. https://doi.org/10.1016/j.ymgme.2013.07.018.

11. Yap S. Classical homocystinuria: newborn screening with early treatment effectively prevents complications. Hamdan Med J. 2012;5:351-362. https://doi.org/10.7707/hmj.v5i3.191.

12. Yap S, Naughten E. Homocystinuria due to cystathionine betasynthase deficiency in Ireland: 25 years' experience of a newborn screened and treated population with reference to clinical outcome and biochemical control. J Inherit Metab Dis. 1998;21:738-747. https://doi.org/10.1023/A:1005445132327.

13. Hall PL, Marquardt G, McHugh DM, et al. Postanalytical tools improve performance of newborn screening by tandem mass spectrometry. Genet Med. 2014;16:889-895. https://doi.org/10.1038/ gim.2014.624262759.

14. McHugh D, Cameron CA, Abdenur JE, et al. Clinical validation of cutoff target ranges in newborn screening of metabolic disorders by tandem mass spectrometry: a worldwide collaborative project. Genet Med. 2011;13:230-254. https://doi.org/10.1097/GIM.0b013 e31820d5e67.

15. Wilson JM, Jungner YG. Principles and practice of mass screening for disease. Bol Oficina Sanit Panam. 1968;65:281-393.

16. Gramer G, Abdoh G, Ben-Omran T, et al. Newborn screening for remethylation disorders and vitamin B12 deficiency-evaluation of new strategies in cohorts from Qatar and Germany. World J Pediatr. 2017;13(2):136-143. https://doi.org/10.1007/s12519-0170003-z.

17. Matern D, Tortorelli S, Oglesbee D, Gavrilov D, Rinaldo P. Reduction of the false-positive rate in newborn screening by implementation of MS/MS-based second-tier tests: the Mayo Clinic experience (2004-2007). J Inherit Metab Dis. 2007;30:585592. https://doi.org/10.1007/s10545-007-0691-y.

18. Okun JG, Gan-Schreier H, Ben-Omran T, et al. Newborn screening for vitamin B6 non-responsive classical Homocystinuria: systematical evaluation of a two-tier strategy. JIMD Rep. 2017;32:8794. https://doi.org/10.1007/8904_2016_556.

19. Tortorelli S, Turgeon CT, Lim JS, et al. Two-tier approach to the newborn screening of methylenetetrahydrofolate reductase deficiency and other remethylation disorders with tandem mass spectrometry. J Pediatr. 2010;157:271-275. https://doi.org/10.1016/j. jpeds.2010.02.027.
20. Turgeon CT, Magera MJ, Cuthbert CD, et al. Determination of total homocysteine, methylmalonic acid, and 2-methylcitric acid in dried blood spots by tandem mass spectrometry. Clin Chem. 2010; 56:1686-1695. https://doi.org/10.1373/clinchem.2010.148957.

21. Malvagia S, Haynes CA, Grisotto L, et al. Heptadecanoylcarnitine (C17) a novel candidate biomarker for newborn screening of propionic and methylmalonic acidemias. Clin Chim Acta. 2015;23: 342-348. https://doi.org/10.1016/j.cca.2015.09.012.

22. Burke Ó, Benton S, Szafranski P, et al. Extending the scope of pooled analyses of individual patient biomarker data from heterogeneous laboratory platforms and cohorts using merging algorithms. Pregnancy Hypertens. 2016;6:53-59. https://doi.org/10. 1016/j.preghy.2015.12.002.

23. Marquardt G, Currier R, McHugh DM, et al. Enhanced interpretation of newborn screening results without analyte cutoff values. Genet Med. 2012;14:648-655. https://doi.org/10.1038/gim.2012.2.

24. Loeber JG, Burgard P, Cornel MC, et al. Newborn screening programmes in Europe; arguments and efforts regarding harmonization. Part 1. From blood spot to screening result. J Inherit Metab Dis. 2012;35:603-611. https://doi.org/10.1007/s10545-012-9483-0.

25. Huemer M, Diodato D, Schwahn B, et al. Guidelines for diagnosis and management of the cobalamin-related remethylation disorders cblC, cblD, cblE, cblF, cblG, cblJ and MTHFR deficiency. J Inherit Metab Dis. 2017;40(1):21-48.

26. Centers for Disease Control and Prevention (CDC) (2017) 2016 quality control program report. Volume 27 , no.2, issued: February 2017

27. Minter Baerg MM, Stoway SD, Hart J, et al. Precision newborn screening for lysosomal disorders. Genet Med. 2017;20:847-854. https://doi.org/10.1038/gim.2017.194.

28. Karaceper MD, Chakraborty P, Coyle D, et al. The health system impact of false positive newborn screening results for mediumchain acyl-CoA dehydrogenase deficiency: a cohort study. Orphanet J Rare Dis. 2016;11:12. https://doi.org/10.1186/s13023-0160391-54741015.

29. Gramer G, Okun JG, Hoffmann GF. Pilot study for evaluation of 21 additional metabolic disorders for the German newborn screening panel. J Inherit Metab Dis. 2016;39:75-83. https://doi.org/10. 1007/s10545-015-9873-1.

30. Röschinger W, Sonnenschein S, Schuhmann E, NennstielRatzel U, Roscher AA, Olgemöller B. New target diseases in newborn screening. Recommendations derived from a pilot study. Monatsschr Kinderheilkd. 2015;2:142-149. https://doi.org/10. 1007/s00112-014-3297-0.

\section{SUPPORTING INFORMATION}

Additional supporting information may be found online in the Supporting Information section at the end of the article.

How to cite this article: Keller R, Chrastina $\mathrm{P}$, Pavlíková M, et al. Newborn screening for homocystinurias: Recent recommendations versus current practice. J Inherit Metab Dis. 2019;42: 128-139. https://doi.org/10.1002/jimd.12034 